\title{
Complexity and approximation of satisfactory partition problems ${ }^{\star}$
}

\author{
Cristina Bazgan ${ }^{1}, Z_{\text {solt Tuza }}{ }^{2}$, and Daniel Vanderpooten ${ }^{1}$ \\ 1 LAMSADE, Université Paris-Dauphine, France \\ \{bazgan, vdp\}@lamsade.dauphine.fr \\ 2 Computer and Automation Institute, Hungarian Academy of Sciences, Budapest \\ and Department of Computer Science, University of Veszprém, Hungary
}

\begin{abstract}
The Satisfactory Partition problem consists of deciding if a given graph has a partition of its vertex set into two nonempty parts such that each vertex has at least as many neighbors in its part as in the other part. This problem was introduced by Gerber and Kobler in 1998 and further studied by other authors but its complexity remained open until now. We prove in this paper that SATisfactory PARTition, as well as a variant where the parts are required to be of the same cardinality, are $N P$-complete. We also study approximation results for the latter problem, showing that it has no polynomial-time approximation scheme, whereas a constant approximation can be obtained in polynomial time. Similar results hold for balanced partitions where each vertex is required to have at most as many neighbors in its part as in the other part.
\end{abstract}

\section{Introduction}

Gerber and Kobler introduced in $[5,6]$ the problem of deciding if a given graph has a vertex partition into two nonempty parts such that each vertex has at least as many neighbors in its part as in the other part. A graph with this property is called satisfactory partitionable. As remarked by Gerber and Kobler, SATISFACTORY PARTITION may have no solution. In particular, the following graphs are not satisfactory partitionable: complete graphs, stars, and complete bipartite graphs with at least one of the two vertex sets having odd size. Some other graphs are easily satisfactory partitionable: cycles of length at least 4, trees which are not stars, and disconnected graphs. After $[5,6]$ this problem was further studied in $[8]$ and [1] but its complexity remained open until now, while some generalizations were studied and proved to be $N P$-complete.

We define in this paper another variant of SATiSfaCtory PARTition, called BALANCED SATISFACTORY PARTition, where the parts are required to have the same cardinality. A graph admitting such a partition is said to be balanced satisfactory partitionable. Graphs like cycles of even length and complete bipartite

\footnotetext{
* This research was supported by the bilateral research cooperation Balaton between EGIDE (France) and Ministry of Education (Hungary) under grant numbers 07244RJ and F-29/2003. The second author was also supported in part by the Hungarian Scientific Research Fund, grant OTKA T-042710.
} 
graphs with both vertex classes of even size are trivially balanced satisfactory partitionable. A graph of even order formed by two non-partitionable connected components of unequal size, however, is an example of a graph which is satisfactory partitionable but not balanced satisfactory partitionable. We show in this paper that Satisfactory Partition and Balanced Satisfactory PartiTION are $N P$-complete.

We consider also the opposite problem of deciding if a given graph has a vertex partition into two parts such that each vertex has at least as many neighbors in the other part as in its own part. This problem called Co-SATisfactory PARTITION corresponds to finding in the graph a maximal cut with respect to moving a vertex from its part to the other. Therefore, a graph always admits such a partition that can be found in polynomial time. However, the balanced version of this problem, called BALANCED Co-SATisfaCtory Partition, does not always admit a solution, e.g. for stars of even order. We prove in this paper that Balanced Co-Satisfactory Partition is NP-complete.

When a graph has no balanced (co-)satisfactory partition, it is natural to ask for a balanced partition maximizing the number of (co-)satisfied vertices. The corresponding optimization problems are MaX SATisfying BALAnced PARtition and MaX Co-Satisfying Balanced Partition. We prove in this paper that Max Satisfying Balanced Partition is 3-approximable, Max Co-Satisfying Balanced Partition is 2-approximable, and that these two problems have no polynomial-time approximation scheme unless $P=N P$.

The paper is structured as follows. Section 2 contains some notations and definitions of problems. In Section 3 we show the $N P$-completeness of SatiSfaCtory Partition, Balanced Satisfactory Partition, and Balanced CoSatisfactory Partition. In Section 4 we prove that Max (Co-)Satisfying Balanced Partition has no approximation scheme, unless $P=N P$, and in Section 5 we give constant approximation algorithms for these problems.

\section{Preliminaries}

We begin with some basic definitions concerning approximation, and then we define the problems considered.

Approximability. Given an instance $x$ of an optimization problem $A$ and a feasible solution $y$ of $x$, we denote by $\operatorname{val}(x, y)$ the value of solution $y$, and by $\operatorname{opt}_{A}(x)$ the value of an optimum solution of $x$. For a function $\rho$, an algorithm is a $\rho$-approximation for a maximization problem $A$ if for any instance $x$ of the problem it returns a solution $y$ such that $\operatorname{val}(x, y) \geq \frac{o p t_{A}(x)}{\rho(|x|)}$. We say that a maximization problem is constant approximable if, for some constant $\rho>1$, there exists a polynomial-time $\rho$-approximation for it. A maximization problem has a polynomial-time approximation scheme (a PTAS, for short) if, for every constant $\varepsilon>0$, there exists a polynomial-time $(1+\varepsilon)$-approximation for it.

Reductions. ([7]) Let $A$ and $A^{\prime}$ be two maximization problems. Then $A$ is said to be gap-preserving reducible to $A^{\prime}$ with parameters $(c, \rho),\left(c^{\prime}, \rho^{\prime}\right)$ (where 
$\rho, \rho^{\prime} \geq 1$ ), if there is a polynomial-time algorithm that transforms any instance $x$ of $A$ to an instance $x^{\prime}$ of $A^{\prime}$ such that the following properties hold:

$$
\operatorname{opt}_{A}(x) \geq c \Rightarrow \operatorname{opt}_{B}\left(x^{\prime}\right) \geq c^{\prime} \quad \text { and } \quad \operatorname{opt}_{A}(x)<\frac{c}{\rho} \Rightarrow \operatorname{opt}_{B}\left(x^{\prime}\right)<\frac{c^{\prime}}{\rho^{\prime}}
$$

Gap-preserving reductions have the following property. If it is $N P$-hard to decide if the optimum of an instance of $A$ is at least $c$ or less than $\frac{c}{\rho}$, then it is $N P$-hard to decide if the optimum of an instance of $A^{\prime}$ is at least $c^{\prime}$ or less than $\frac{c^{\prime}}{\rho^{\prime}}$. This $N P$-hardness implies that $A^{\prime}$ is hard to $\rho^{\prime}$-approximate.

Graphs. We consider finite, undirected graphs without loops and multiple edges. For a graph $G=(V, E)$, a vertex $v \in V$, and a subset $Y \subseteq V$ we denote by $d_{Y}(v)$ the number of vertices in $Y$ that are adjacent to $v$; and, as usual, we write $d(v)$ for the degree $d_{V}(v)$ of $v$ in $V$. A partition $\left(V_{1}, V_{2}\right)$ of $V$ is said to be nontrivial if both $V_{1}$ and $V_{2}$ are nonempty.

The problems we are interested in are defined as follows.

\section{SATISFACTORY PARTITION}

Input: A graph $G=(V, E)$.

Question: Is there a nontrivial partition $\left(V_{1}, V_{2}\right)$ of $V$ such that for every $v \in V$, if $v \in V_{i}$ then $d_{V_{i}}(v) \geq d_{V_{3-i}}(v)$ ?

The variant of this problem where the two parts have equal size is:

Balanced Satisfactory Partition

Input: A graph $G=(V, E)$ on an even number of vertices.

Question: Is there a partition $\left(V_{1}, V_{2}\right)$ of $V$ such that $\left|V_{1}\right|=\left|V_{2}\right|$ and for every $v \in V$, if $v \in V_{i}$ then $d_{V_{i}}(v) \geq d_{V_{3-i}}(v)$ ?

Given a partition $\left(V_{1}, V_{2}\right)$, we say that a vertex $v \in V_{i}$ is satisfied if $d_{V_{i}}(v) \geq$ $d_{V_{3-i}}(v)$, or equivalently if $d_{V_{i}}(v) \geq\left\lceil\frac{d(v)}{2}\right\rceil$. A graph admitting a nontrivial partition where all vertices are satisfied is called satisfactory partitionable, and such a partition is called a satisfactory partition. If $\left|V_{1}\right|=\left|V_{2}\right|$ also holds, then it will be called a balanced satisfactory partition and the graph $G$ is balanced satisfactory partitionable.

Co-SATISFACTORY PARTition

Input: A graph $G=(V, E)$.

Question: Is there a partition $\left(V_{1}, V_{2}\right)$ of $V$ such that for every $v \in V$, if $v \in V_{i}$ then $d_{V_{i}}(v) \leq d_{V_{3-i}}(v)$ ?

We already mentioned in the introduction that Co-Satisfactory PARTITION always has a solution which can be found easily in polynomial time.

Balanced Co-Satisfactory Partition

Input: A graph $G=(V, E)$ on an even number of vertices.

Question: Is there a partition $\left(V_{1}, V_{2}\right)$ of $V$ such that $\left|V_{1}\right|=\left|V_{2}\right|$ and for every $v \in V$, if $v \in V_{i}$ then $d_{V_{i}}(v) \leq d_{V_{3-i}}(v)$ ?

Given a partition $\left(V_{1}, V_{2}\right)$, a vertex $v \in V_{i}$ is co-satisfied if $d_{V_{i}}(v) \leq d_{V_{3-i}}(v)$, or equivalently if $d_{V_{i}}(v) \leq\left\lceil\frac{d(v)}{2}\right\rceil$. The previous notions are similarly defined for co-satisfiability. 
When a graph is not balanced (co-)satisfactory partitionable, it is natural to ask for a balanced partition that maximizes the number of vertices that are (co-)satisfied. Therefore, we consider the following problems.

Max SATisfying BALANCEd PARTition

Input: A graph $G=(V, E)$ on an even number of vertices.

Output: A partition $\left(V_{1}, V_{2}\right)$ of $V$, such that $\left|V_{1}\right|=\left|V_{2}\right|$, that maximizes the number of satisfied vertices.

Max Co-Satisfying Balanced Partition

Input: A graph $G=(V, E)$ on an even number of vertices.

Output: A partition $\left(V_{1}, V_{2}\right)$ of $V$, such that $\left|V_{1}\right|=\left|V_{2}\right|$, that maximizes the number of co-satisfied vertices.

Almost Balanced Partitions. The above problems can also be formulated for graphs with an odd number of vertices, requiring partitions $\left(V_{1}, V_{2}\right)$ such that $\left|V_{1}\right|$ and $\left|V_{2}\right|$ differ just by 1 .

\section{Complexity of (Balanced) (Co-)Satisfactory Partition}

In this section we establish the NP-completeness of the following three problems:

(i) SAtisfactory Partition

(ii) Balanced Satisfactory Partition

(iii) Balanced Co-Satisfactory Partition

The overall scheme is that (iii) is $N P$-complete, (iii) is reducible to (ii), and (ii) is reducible to (i).

Proposition 1. BALANCED SATISFACTORY PARTITION is polynomial-time reducible to SATISFACTORY PARTition.

Proof. Let $G=(V, E)$ be a graph, instance of the first problem on $n$ vertices. The graph $G^{\prime}=\left(V^{\prime}, E^{\prime}\right)$, instance of SAtisfactory PARTition, is obtained from $G$ by adding two cliques of size $\frac{n}{2}, A=\left\{a_{1}, \ldots, a_{\frac{n}{2}}\right\}$ and $B=\left\{b_{1}, \ldots, b_{\frac{n}{2}}\right\}$. In $G^{\prime}$, in addition to the edges of $G$, all vertices of $V$ are adjacent to all vertices of $A$ and $B$. Also each vertex $a_{i} \in A$ is linked to all vertices of $B$ except $b_{i}$, $i=1, \ldots, \frac{n}{2}$.

Let $\left(V_{1}, V_{2}\right)$ be a balanced satisfactory partition of $G$. Then $\left(V_{1}^{\prime}, V_{2}^{\prime}\right)$ where $V_{1}^{\prime}=V_{1} \cup A$ and $V_{2}^{\prime}=V_{2} \cup B$ is a satisfactory partition of $G^{\prime}$. Indeed, a vertex from $A \cup B$ is satisfied, for example if $v \in A, d_{V_{1}^{\prime}}(v)=\left|V_{1}\right|+|A|-1=\left|V_{2}\right|+|B|-1=$ $d_{V_{2}^{\prime}}(v)$. Also it is easy to see that a vertex from $V$ is satisfied in $G^{\prime}$ since it is satisfied in $G$.

Let $\left(V_{1}^{\prime}, V_{2}^{\prime}\right)$ be a satisfactory partition of $G^{\prime}$, where $V_{1}^{\prime}=V_{1} \cup A_{1} \cup B_{1}$ and $V_{2}^{\prime}=V_{2} \cup A_{2} \cup B_{2}$ with $V_{i} \subseteq V, A_{i} \subseteq A, B_{i} \subseteq B, i=1,2$. We claim that $\left(V_{1}, V_{2}\right)$ is a balanced satisfactory partition of $G$.

We first show that $A_{1} \cup B_{1} \neq \emptyset$ and $A_{2} \cup B_{2} \neq \emptyset$, which means that no satisfactory partition can contain $A \cup B$ in one of its parts. Indeed, by contradiction, suppose we have $V_{1}^{\prime}=V_{1} \cup A \cup B$ and $V_{2}^{\prime}=V_{2}$. Then, the inequality specifying that $v \in V_{2}$ is satisfied is $d_{V_{2}}(v) \geq d_{V_{1}}(v)+n$ which is impossible. So, 
two cases are possible: either each part of the partition contains one clique, say $V_{1}^{\prime}=V_{1} \cup A$ and $V_{2}^{\prime}=V_{2} \cup B$ (case 1 ) or at least one of the cliques is cut by the partition (case 2).

In case 1 , in order for a vertex of $A$ to be satisfied, we have $\frac{n}{2}-1+\left|V_{1}\right| \geq$ $\left|V_{2}\right|+\frac{n}{2}-1$ and in order that a vertex of $B$ be satisfied, we have $\frac{n}{2}-1+\left|V_{2}\right| \geq$ $\left|V_{1}\right|+\frac{n}{2}-1$. These two inequalities imply $\left|V_{1}\right|=\left|V_{2}\right|$. Moreover, since $v \in V_{1} \cup V_{2}$ is satisfied in $G^{\prime}$ where it is linked to $\frac{n}{2}$ vertices in $A$ and $\frac{n}{2}$ vertices in $B, v$ is also satisfied in $G$.

In case 2 , suppose that clique $A$ is cut by the partition into non-empty sets $A_{1}$ and $A_{2}$ while $B_{1}$ or $B_{2}$ may be empty. We show now that if $a_{i} \in A_{1}$ for some $i$, then also $b_{i} \in B_{2}$ for the same $i$. Assume by contradiction that $b_{i} \in B_{1}$. Since $a_{i}$ is satisfied we have

This implies $\left|V_{1}^{\prime}\right|>\left|V_{2}^{\prime}\right|$.

$$
\left(\left|A_{1}\right|-1\right)+\left(\left|B_{1}\right|-1\right)+\left|V_{1}\right| \geq\left|A_{2}\right|+\left|B_{2}\right|+\left|V_{2}\right|
$$

Let $a_{j} \in A_{2}$. We may have $b_{j} \in B_{1}$ or $b_{j} \in B_{2}$. If $b_{j} \in B_{2}$ then the condition that $a_{j}$ is satisfied is

$$
\left(\left|A_{2}\right|-1\right)+\left(\left|B_{2}\right|-1\right)+\left|V_{2}\right| \geq\left|A_{1}\right|+\left|B_{1}\right|+\left|V_{1}\right|
$$

If $b_{j} \in B_{1}$ then the condition that $a_{j}$ is satisfied is

$$
\left(\left|A_{2}\right|-1\right)+\left|B_{2}\right|+\left|V_{2}\right| \geq\left|A_{1}\right|+\left(\left|B_{1}\right|-1\right)+\left|V_{1}\right|
$$

Each of (2) and (3) implies that $\left|V_{2}^{\prime}\right| \geq\left|V_{1}^{\prime}\right|$, contradicting (1). Thus $\left|A_{1}\right|=$ $\left|B_{2}\right|$ and $\left|A_{2}\right|=\left|B_{1}\right|$, that means that both cliques are cut by the partition.

For $a_{i} \in A_{1}$ and $b_{i} \in B_{2}$ the inequalities specifying that $a_{i}$ and $b_{i}$ are satisfied are respectively:

and

$$
\begin{aligned}
& \left(\left|A_{1}\right|-1\right)+\left|B_{1}\right|+\left|V_{1}\right| \geq\left|A_{2}\right|+\left(\left|B_{2}\right|-1\right)+\left|V_{2}\right| \\
& \left|A_{2}\right|+\left(\left|B_{2}\right|-1\right)+\left|V_{2}\right| \geq\left(\left|A_{1}\right|-1\right)+\left|B_{1}\right|+\left|V_{1}\right|
\end{aligned}
$$

from which we obtain $\left|A_{1}\right|+\left|B_{1}\right|+\left|V_{1}\right|=\left|A_{2}\right|+\left|B_{2}\right|+\left|V_{2}\right|$. Since $\left|A_{1}\right|=\left|B_{2}\right|$ and $\left|A_{2}\right|=\left|B_{1}\right|$, we get $\left|V_{1}\right|=\left|V_{2}\right|$.

Moreover, since $v \in V_{1} \cup V_{2}$ is satisfied in $G^{\prime}$ where it is linked to $\left|A_{1}\right|+\left|B_{1}\right|=$ $\frac{n}{2}$ vertices in $V_{1}^{\prime}$ among the vertices of the two cliques and $\left|A_{2}\right|+\left|B_{2}\right|=\frac{n}{2}$ vertices in $V_{2}^{\prime}, v$ is also satisfied in $G$.

We state now our NP-completeness results.

Theorem 1. Balanced Co-Satisfactory Partition is NP-complete.

Proof. Clearly, this problem is in NP. We construct a polynomial reduction from a variant of INDEPENDENT SET, the problem of deciding if a graph with $n$ vertices contains an independent set of size at least $\frac{n}{2}$, a problem stated to be $N P$-hard in [4]. Let $G=(V, E)$ be a graph with $n$ vertices $v_{1}, \ldots, v_{n}$ and $m$ edges, an input of this variant of InDEPENDENT SET problem. We assume that $n$ is even, since otherwise we can add a vertex that we link with all the vertices of the graph without changing the problem. The edges of $G$ are labelled $e_{1}, \ldots, e_{m}$. We construct a graph $G^{\prime}=\left(V^{\prime}, E^{\prime}\right)$, instance of BALANCED CoSATisfactory PARTition as follows: the vertex set $V^{\prime}$ consists of three sets $F, T$ and $V$ (the vertex set of $G$ ) where $F=\left\{f_{1}, \ldots, f_{2 m+1}\right\}$ and $T=\left\{t_{1}, \ldots, t_{2 m+1}\right\}$. Vertices $f_{2 \ell}, f_{2 \ell+1}$ correspond to edge $e_{\ell}(\ell=1, \ldots, m)$ and $f_{1}$ is an additional vertex. $F$ and $T$ are two independent sets of size $2 m+1$. Vertices $t_{i}$ are linked 
with $f_{j}, i=1, \ldots, 2 m+1, j=1, \ldots, 2 m+1$. In addition to these edges and $E$, the edge set $E^{\prime}$ contains the edges $\left(f_{2 \ell}, v_{i}\right)$ and $\left(f_{2 \ell+1}, v_{j}\right)$ for each edge $e_{\ell}=\left(v_{i}, v_{j}\right), \ell=1, \ldots, m$.

It is easy to see that this construction can be accomplished in polynomial time. All that remains to show is that $G$ has an independent set of size at least $\frac{n}{2}$ if and only if $G^{\prime}$ is balanced co-satisfactory partitionable.

Suppose firstly that $G$ has an independent set of size at least $\frac{n}{2}$. Let $S$ be an independent set of size exactly $\frac{n}{2}$ of $G$. Let $V_{1}^{\prime}=F \cup S$ and $V_{2}^{\prime}=T \cup \bar{S}$, where $\bar{S}=V \backslash S$. Let us check in the following that $\left(V_{1}^{\prime}, V_{2}^{\prime}\right)$ is a balanced cosatisfactory partition. It is easy to see that all vertices of $F$ and $T$ are co-satisfied. Let $v \in S$. Since $S$ is an independent set, $v$ is not linked to any vertex in $S$. Thus, $d_{V_{1}^{\prime}}(v)=d_{\bar{S}}(v)=d_{V_{2}^{\prime}}(v)$ and so the vertices of $S$ are co-satisfied. Given a vertex $v \in \bar{S}, d_{V_{1}^{\prime}}(v)=2 d_{S}(v)+d_{\bar{S}}(v)$ while $d_{V_{2}^{\prime}}(v)=d_{\bar{S}}(v)$, thus also the vertices of $\bar{S}$ are co-satisfied in $G^{\prime}$.

Suppose now that $G^{\prime}$ is balanced co-satisfactory partitionable and let $\left(V_{1}^{\prime}, V_{2}^{\prime}\right)$ be a balanced co-satisfactory partition. It is easy to see that $F$ and $T$ cannot be both included in the same part of the partition since otherwise the vertices of $F$ and $T$ are not co-satisfied. If the partition cuts only one of the two sets $F$ or $T$, suppose for example that $F$ is cut, then the vertices of $F$ that are in the same part of the partition as $T$ are not co-satisfied. If the partition cuts both $F$ and $T$, denote by $F_{1}, T_{1}$ and $F_{2}, T_{2}$ the sets of vertices of $F$ and $T$ that are included in $V_{1}^{\prime}$ and $V_{2}^{\prime}$ respectively. For vertices of $T_{1}$ to be co-satisfied, we first have $\left|F_{1}\right| \leq\left|F_{2}\right|$ whereas for vertices of $T_{2}$ to be co-satisfied, we must have $\left|F_{2}\right| \leq\left|F_{1}\right|$, that is $\left|F_{1}\right|=\left|F_{2}\right|$, which is impossible since $|F|$ is odd. Therefore, $F$ and $T$ are included in different parts of the partition and thus $\left(V_{1}^{\prime}, V_{2}^{\prime}\right)$ cuts the set $V$ into two balanced sets $V_{1}, V_{2}$, where $V_{1}^{\prime}=F \cup V_{1}$ and $V_{2}^{\prime}=T \cup V_{2}$. We show that $V_{1}$ is an independent set. A vertex $v \in V_{1}$ has $d_{V_{1}^{\prime}}(v)=2 d_{V_{1}}(v)+d_{V_{2}}(v)$ and $d_{V_{2}^{\prime}}(v)=d_{V_{2}}(v)$. Since $v$ is co-satisfied in $G^{\prime}$ we have $d_{V_{1}^{\prime}}(v) \leq d_{V_{2}^{\prime}}(v)$ and we obtain that $d_{V_{1}}(v)=0$. Thus $V_{1}$ is an independent set of size $\frac{n}{2}$.

Theorem 2. SATisfactory Partition and BAlAnCEd Satisfactory ParTITION are NP-complete.

Proof. Clearly, these two problems are in NP. We reduce BALANCED Co-SatisFactory Partition to Balanced Satisfactory Partition which shows the $N P$-completeness of the latter problem by Theorem 1 . Proposition 1 implies the $N P$-completeness of SATISFACTORY PARTITION. The reduction is as follows.

Let $G$ be a graph, instance of BALANCED Co-SAtisfactory Partition on $n$ vertices $v_{1}, \ldots, v_{n}$. The graph $G^{\prime}$, instance of BALANCED SATISFACTORY PARTition, has $2 n$ vertices $v_{1}, \ldots, v_{n}, u_{1}, \ldots, u_{n} . G^{\prime}$ is the complement of graph $G$ on vertices $v_{1}, \ldots, v_{n}$, and we add pendant edges $\left(u_{i}, v_{i}\right), i=1, \ldots, n$. If $G$ is balanced co-satisfactory partitionable and $\left(V_{1}, V_{2}\right)$ is such a partition, then $V_{i}^{\prime}=V_{i} \cup\left\{u_{j}: v_{j} \in V_{i}\right\}$ is a balanced satisfactory partition for $G^{\prime}$. Indeed, if $v_{i} \in V_{1}$ then $d_{V_{1}}\left(v_{i}\right) \leq d_{V_{2}}\left(v_{i}\right)$ in $G$. Thus, in $G^{\prime}$ we have $d_{V_{1}^{\prime}}\left(v_{i}\right)=\frac{n}{2}-1-$ $d_{V_{1}}\left(v_{i}\right)+1 \geq \frac{n}{2}-d_{V_{2}}\left(v_{i}\right)=d_{V_{2}^{\prime}}\left(v_{i}\right)$ and $d_{V_{1}^{\prime}}\left(u_{i}\right)=1>d_{V_{2}^{\prime}}\left(u_{i}\right)=0$. Conversely, since in each balanced satisfactory partition of $G^{\prime}, u_{i}$ is in the same set as $v_{i}$, such a partition of $G^{\prime}$ gives a balanced co-satisfactory partition in $G$. 


\section{No PTAS for Max (Co-)Satisfying Balanced Partition}

In this section we prove that MAX Co-SAtisfying BALANCED PARtition and Max SATisfying BAlanced PARTition have no polynomial-time approximation scheme unless $P=N P$. We first introduce a problem used in our reductions.

MAX $k$-Vertex COVER-B

Input: A graph $G=(V, E)$ with $|V| \geq k$ and maximum degree at most $B$.

Output: The maximum number of edges in $G$ that can be covered by a subset $V^{\prime} \subseteq V$ of cardinality $k$.

Theorem 3 (Petrank [7]). There exists a constant $\alpha, 0<\alpha<1$ with the following property: given a graph $G$ with $n$ vertices and $m$ edges, instance of MAX $k$-Vertex COVER-B for some $k=\Theta(n)$, it is NP-hard to distinguish, whether it has opt $(G)=m$ or opt $(G)<(1-\alpha) m$.

Though it is not explicitly mentioned in [7], the proof of Theorem 3 yields the same conclusion for the restricted class of graphs with $m \geq \frac{n}{2}$. We prove next that the previous result holds in particular for $k=\frac{n}{2}$.

Theorem 4. There exists a constant $\beta, 0<\beta<1$, with the following property: given a graph $G$ with $N$ vertices and $M$ edges, instance of MAX $\frac{N}{2}$-VERTEX Cover-B', it is NP-hard to distinguish whether it has opt $(G)=M \operatorname{or} \operatorname{opt}(G)<$ $(1-\beta) M$.

Proof. We construct a gap-preserving reduction from MAX $k$-VERTEx CoverB with $k=c n$, for some constant $c<1$, to MAX $\frac{N}{2}$-Vertex Cover- $(2 \mathrm{~B}+2)$. Let $G=(V, E)$ be a graph on $n$ vertices and $m \geq \frac{n}{2}$ edges, instance of MAX $k$-Vertex Cover-B. We will construct a graph $G^{\prime \prime}$ with $N$ vertices and $M$ edges such that if $\operatorname{opt}(G)=m$ then $\operatorname{opt}\left(G^{\prime \prime}\right)=M$ and if $\operatorname{opt}(G) \leq(1-\alpha) m$, for some $\alpha>0$, then $\operatorname{opt}\left(G^{\prime \prime}\right) \leq(1-\beta) M$, for some $\beta>0$.

First assume that $c>1 / 2$. Let $G^{\prime \prime}$ be the graph obtained from $G$ by inserting $2 k-n$ isolated vertices. In this case, the properties of the gap-preserving reduction hold with $\beta=\alpha$.

Consider now the case $c<1 / 2$. Suppose first that $n-2 k$ is a multiple of $B+1$. Let $G^{\prime \prime}$ be the graph that consists of a copy of $G$ and $\frac{n-2 k}{B+1}$ copies of the graph $T_{B+1}$ which is the complete tripartite graph whose vertex classes have cardinality $B+1$ each. Observe that $T_{B+1}$ needs $2 B+2$ vertices in covering its edges (the complement of a vertex class), and if just $2 B+2-t$ vertices are taken, then at least $t(B+1)$ edges remain uncovered. Thus, since $G$ has maximum degree at most $B$, each subset of $\frac{N}{2}$ vertices not covering all copies of $T_{B+1}$ is trivially improvable. Suppose first that $\operatorname{opt}(G)=m$ and let $V^{\prime}$ be a vertex cover of size $k$ in $G$. Then the set $V^{\prime}$ and the vertices of two among the 3 independent sets of each of the $\frac{n-2 k}{B+1}$ copies of $T_{B+1}$ form a vertex cover of $G^{\prime \prime}$ of size $\frac{N}{2}$, and thus $\operatorname{opt}\left(G^{\prime \prime}\right)=M$. On the other hand, suppose opt $(G)<(1-\alpha) m$. Then since $M=m+3(B+1)(n-2 k)$ and $m \geq \frac{n}{2}$, the number of edges not covered in $G^{\prime \prime}$ is at least $\alpha m \geq \frac{\alpha M}{1+6(B+1)(1-2 c)}$ that can be viewed as $\beta M$. 
Finally, if $c<1 / 2$ and if $n-2 k=\ell \bmod (B+1), 0<\ell \leq B$, then let $G^{\prime}$ be the graph $G$ together with further $B+1-\ell$ isolated vertices. Now, we can transform $G^{\prime}$ to $G^{\prime \prime}$ as before by inserting $\frac{n-2 k-\ell}{B+1}+1$ copies of $T_{B+1}$. In this case we get a slightly different value for $\beta$, as the number $m$ of edges is now compared with the modified number $n+B+1-\ell$ of vertices. Nevertheless, $\beta>0$ is obtained.

From this theorem, the following non-approximability results can be deduced.

Theorem 5. MAX Co-SATisfying BAlanced PARTition has no polynomialtime approximation scheme unless $P=N P$.

Proof. We construct a gap-preserving reduction between MAX $\frac{n}{2}$-VERTEX CoverB and Max Co-Satisfying Balanced Partition. Let $G$ be a graph instance of MAX $\frac{n}{2}$-VERTEX COVER-B on $n$ vertices and $m$ edges. We construct the graph $G^{\prime}$ as in the proof of Theorem 1. Denote by $N$ the number of vertices of $G^{\prime}$.

Suppose first that $\operatorname{opt}(G)=m$, and let $V^{\prime}$ be a vertex cover of size $\frac{n}{2}$ of $G$. Then in the partition $\left(F \cup\left(V \backslash V^{\prime}\right), T \cup V^{\prime}\right)$ all vertices are co-satisfied and thus $\operatorname{opt}\left(G^{\prime}\right)=N$.

Suppose now that $\operatorname{opt}(G)<(1-\beta) m$. Thus for any set of $\frac{n}{2}$ vertices $V^{\prime}$, at least $\beta m$ edges of $G$ remain uncovered. The number of vertices incident to a non-covered edge is at least $\frac{2 \beta m}{B}$. These vertices are not co-satisfied in the partition $\left(F \cup\left(V \backslash V^{\prime}\right), T \cup V^{\prime}\right)$ and thus the number of co-satisfied vertices in this partition is less than $N-\frac{2 \beta m}{B}$. It is lengthy but not too hard to show that, when a balanced partition cuts $F$ or/and $T$, at least $\mathrm{cm}$ vertices are not co-satisfied, for some constant $c<1$, and thus in this case we have $\operatorname{opt}\left(G^{\prime}\right)<N-d m$, for some constant $d$. Since MAX $k$-Vertex Cover-B is trivial for $m \leq k$, we may assume that $m \geq \frac{n}{2}$. Thus, since the number of vertices of $G^{\prime}, N=4 m+2+n \leq 7 m$, we obtain $\operatorname{opt}\left(G^{\prime}\right)<\left(1-\frac{d}{7}\right) N$.

Theorem 6. MAX SATISFying BALANCEd PARTition has no polynomial-time approximation scheme unless $P=N P$.

Proof. Consider the graph $G^{\prime}$ with $N$ vertices and $M$ edges obtained in the construction given in the proof of Theorem 5, and apply to $G^{\prime}$ the reduction given in Theorem 2. Let $G^{\prime \prime}$ be the graph obtained. It can be shown that if $\operatorname{opt}\left(G^{\prime}\right)=N$ then $\operatorname{opt}\left(G^{\prime \prime}\right)=2 N$ and if $\operatorname{opt}\left(G^{\prime}\right)<(1-\gamma) N$ then $\operatorname{opt}\left(G^{\prime \prime}\right)<2 N(1-c \gamma)$ for some constant $c$.

\section{Constant approximations for Max (Co-)Satisfying Balanced Partition}

We concentrate mostly on the approximation of MAX SATISFYING BALANCED PARTITION. The co-satisfying version turns out to be simpler, and will be considered at the end of the section.

Proposition 2. Any graph $G$ with an odd number of vertices $n$ has an almost balanced partition such that each vertex in the part of size $\frac{n+1}{2}$ is satisfied, and such a partition can be found in polynomial time. 
Proof. Let $\left(V_{1}, V_{2}\right)$ be an almost balanced partition of $G$ with $\left|V_{1}\right|>\left|V_{2}\right|$. If $V_{1}$ contains a vertex $v$ that is not satisfied, then $d_{V_{1}}(v)<d_{V_{2}}(v)$ and thus by moving $v$ from $V_{1}$ to $V_{2}$ we obtain an almost balanced partition with a smaller value of the cut induced by $\left(V_{1}, V_{2}\right)$. The algorithm repeats this step while the largest set contains a non-satisfied vertex. After at most $|E|$ steps we obtain an almost balanced partition where the largest set contains only satisfied vertices.

We consider now graphs of even order. Given a graph on an even number of vertices $n$, a vertex of degree $n-1$ is never satisfied in a balanced partition since it has only $\frac{n}{2}-1$ neighbors in its own part and $\frac{n}{2}$ neighbors in the other part.

Theorem 7. In any graph $G$ on an even number of vertices $n$, a balanced partition with at least $\left\lceil\frac{n-t}{3}\right\rceil$ satisfied vertices can be found in polynomial time, where $t$ is the number of vertices of degree $n-1$ in $G$.

Proof. If $G$ is not connected, then we find an almost balanced partition in each odd connected component, using Proposition 2, and a balanced partition in each even connected component (as shown afterwards); and then it is easy to put them together in order to form a balanced partition of $G$, where at least $\left\lceil\frac{n-t}{3}\right\rceil$ vertices are satisfied.

Suppose in the following that $G$ is connected, and let $H$ be the complement of $G$. Let $H_{1}, \ldots, H_{q}(q \geq 1)$ be the connected components of $H$. Observe that if a vertex is of degree $n-1$ in $G$ then it forms alone a connected component in $H$. Denote by $n_{i}$ the number of vertices of $H_{i}, i=1, \ldots, q$. Consider now a connected component $H_{i}$, where $n_{i}>1$. We will show that a (almost) balanced partition of $V\left(H_{i}\right)$ can be constructed where at least $\left\lceil\frac{n_{i}}{3}\right\rceil$ vertices are satisfied in $G$. (For $n_{i}$ odd and $n$ even, the almost balanced partition found in Proposition 2 may not work, since its smaller part will be completed with too many, $\frac{n}{2}-\frac{n_{i}-1}{2}$ vertices in $G$.)

Let $M=\left\{\left(a_{1}, b_{1}\right), \ldots,\left(a_{p}, b_{p}\right)\right\}$ be a maximum matching in $H_{i}$. It can be found efficiently, using e.g. Edmonds' algorithm [2]. We distinguish two cases.

If $|M| \geq\left\lceil\frac{n_{i}}{3}\right\rceil$ then consider a (almost) balanced partition of the vertices of $V\left(H_{i}\right)$ except the vertices of the matching $M$. Let $\left(V_{1}, V_{2}\right)$ be the partition of $V\left(H_{i}\right)$ obtained from this one by adding vertices $a_{j}$ to $V_{1}$ and vertices $b_{j}$ to $V_{2}$. While there exists a pair $\left(a_{j}, b_{j}\right)$ where both vertices are not satisfied (in $G$ ), we exchange these two vertices. Since $a_{j}$ and $b_{j}$ are not linked in $G$, this exchange makes both $a_{j}$ and $b_{j}$ satisfied and decreases the value of the cut by at least 2 . Therefore, after at most $\frac{|E|}{2}$ exchanges, we obtain a (almost) balanced partition with at least $\left\lceil\frac{n_{i}}{3}\right\rceil$ vertices satisfied (at least one vertex in each pair $\left(a_{j}, b_{j}\right)$ ).

If $|M|<\left\lceil\frac{n_{i}}{3}\right\rceil$ then using Gallai's decomposition theorem [3] we can obtain in polynomial time a vertex set $S$ such that $2|M|=n_{i}-\ell+|S|$ where $\ell$ is the number of odd connected components of $H_{i}-S$. Let $O_{1}, \ldots, O_{\ell}$ be the odd connected components of $H_{i}-S$. Thus $\ell-|S| \geq\left\lceil\frac{n_{i}}{3}\right\rceil$ and so $\ell \geq\left\lceil\frac{n_{i}}{3}\right\rceil$ and $|S| \leq\left\lceil\frac{n_{i}}{3}\right\rceil$. Let us consider a vertex $v_{j} \in O_{j}$ linked to a vertex of $S$, for $j=1, \ldots, \ell$. Those $v_{j}$ are mutually adjacent in $G$.

If $\ell \geq\left\lceil\frac{n_{i}}{2}\right\rceil$ then we consider the following (almost) balanced partition $\left(V_{1}, V_{2}\right)$ : $V_{1}$ contains $\left\lceil\frac{n_{i}}{2}\right\rceil$ vertices from $v_{1}, \ldots, v_{\ell}$ and $V_{2}$ contains the other vertices. It 
is easy to see that at least $\left\lceil\frac{n_{i}}{2}\right\rceil$ vertices are satisfied in $G$, since for $v_{j} \in V_{1}$ we have $d_{V_{1}}\left(v_{j}\right)=\left\lceil\frac{n_{i}}{2}\right\rceil-1$ and $d_{V_{2}}\left(v_{j}\right) \leq\left\lfloor\frac{n_{i}}{2}\right\rfloor-1$.

Suppose next that $\left\lceil\frac{n_{i}}{3}\right\rceil \leq \ell \leq\left\lceil\frac{n_{i}}{2}\right\rceil$. If $n_{i}$ is even, we construct a balanced partition $\left(V_{1}^{\prime}, V_{2}^{\prime}\right)$ where $V_{1}^{\prime}$ contains $v_{1}, \ldots, v_{\ell}$ and $V_{2}^{\prime}$ contains $S$ and $\ell-|S|$ other vertices; and if $n_{i}$ is odd, we construct an almost balanced partition $\left(V_{1}^{\prime}, V_{2}^{\prime}\right)$ where $V_{1}^{\prime}$ contains $v_{1}, \ldots, v_{\ell}$ and $V_{2}^{\prime}$ contains $S$ and $\ell-1-|S|$ other vertices. In this latest step we pay attention, when we take some vertices from the remaining vertices of an odd connected component $O_{j}$, to take always an even number of vertices. In order to obtain a (almost) balanced partition $\left(V_{1}, V_{2}\right)$ from $\left(V_{1}^{\prime}, V_{2}^{\prime}\right)$ we consider the remaining vertices of each odd connected component $O_{j}$ and we put half of these vertices in $V_{1}$ and half in $V_{2}$ such that $v_{j}$ is satisfied. The partition in $O_{j}$ does not influence the satisfied status of $v_{s}$ for $s \neq j$, therefore it can be done independently in all $O_{j}$. We complete this partition by putting half of the remaining vertices in $V_{1}$ and half in $V_{2}$.

Theorem 8. MAX SATisfying BALAnCEd PARTition is 3-approximable.

Proof. Given a graph on $n$ vertices, the maximum number of vertices that are satisfied in a balanced partition is $\operatorname{opt}(G) \leq n-t$, where $t$ is the number of vertices of degree $n-1$. Using Theorem 7 we obtain in polynomial time a balanced partition where the number of satisfied vertices is $\mathrm{val} \geq\left\lceil\frac{n-t}{3}\right\rceil \geq \frac{\operatorname{opt}(G)}{3}$.

Theorem 9. Max Co-Satisfying Balanced Partition is 2-approximable.

Proof. Let $\left(V_{1}, V_{2}\right)$ be a balanced partition of $G$. While there exists $v_{1} \in V_{1}$ and $v_{2} \in V_{2}$ that are not co-satisfied, we exchange $v_{1}$ and $v_{2}$. After this exchange the value of the cut increases by at least 2 . Thus, after $\frac{|E|}{2}$ steps we obtain a balanced partition where at least one of the two parts contains co-satisfied vertices only.

\section{References}

1. C. Bazgan, Zs. Tuza and D. Vanderpooten, On the existence and determination of satisfactory partitions in a graph, Proc. ISAAC 2003, LNCS 2906, 444-453.

2. J. Edmonds, Paths, trees, and flowers, Canadian J. of Math. 17 (1965), 449-467.

3. T. Gallai, Maximale Systeme unabhängiger Kanten, Magyar Tud. Akad. Mat. Kutató Int. Közl. 9 (1964), 401-413.

4. M. R. Garey and D. S. Johnson, Computers and Intractability: A Guide to the Theory of NP-Completeness, W. H. Freeman and Co., San Francisco, 1979.

5. M. Gerber and D. Kobler, Partitioning a graph to satisfy all vertices, Technical report, Swiss Federal Institute of Technology, Lausanne, 1998.

6. M. Gerber and D. Kobler, Algorithmic approach to the satisfactory graph partitioning problem, European Journal of Operation Research 125 (2000), 283-291.

7. E. Petrank, The hardness of approximation: gap location, Computational Complexity 4 (1994), 133-157.

8. K. H. Shafique and R. D. Dutton, On satisfactory partitioning of graphs, Congressus Numerantium 154 (2002), 183-194. 